\title{
Divulgación como Mecanismo para prevenir la Falla de Mercado
}

Disclosure as a Mechanism to prevent Market Failure

Prof. Dr. Florencia Marotta-Wurgler, PhD

Professor of Law New York University

Traducción

RFJ, No. 2, 2017, pp. 411-436, ISSN 2588-0837

RESUMEN: los reguladores a menudo abordan el potencial de los contratos de formulario estándar unilateral requiriendo una divulgación adicional de los términos. A pesar de su ubicuidad, para que un régimen de divulgación sea efectivo, debe aumentar el número de lectores de los contratos más allá de una tasa no trivial, $\mathrm{y}$, además, las personas deben estar dispuestas a cambiar sus decisiones condicionadas a lo que leen. Sigo el flujo de clics de 47,399 hogares a 81 minoristas de software de Internet para probar si quienes compran software en línea son más propensos a leer el acuerdo de licencia cuando se divulga de manera más destacada. Encuentro que el grado de divulgación casi no tiene impacto en la tasa a la que los consumidores leen los acuerdos de licencia. Además, aquellos que sí lo hacen tienen la misma probabilidad de comprar el producto de software, independientemente de la parcialidad del contrato. Los resultados sugieren que exigir la divulgación en línea es poco probable, por sí solo, para presionar a los vendedores.

PALABRAS CLAVES: fallas del mercado, divulgación de información, mercado, productos tecnológicos , consumidores.

ABSTRACT: regulators often address the potential for one-sided standard form contracts by requiring extra disclosure of the terms. Despite its ubiquity, for a disclosure regime to be effective, it must increase readership of contracts beyond a nontrivial rate, and, in addition, individuals must be willing to change their decisions conditional on what they read. I follow the clickstream of 47,399 households to 81 Internet software retailers to test whether those who shop for software online are more likely to read the license agreement 
when it is more prominently disclosed. I find that the degree of disclosure has almost no impact on the rate at which consumers read license agreements. Moreover, those who do read are equally likely to purchase the software product regardless of the one-sidedness of the contract. The results suggest that mandating disclosure online is unlikely, on its own, to put pressure on sellers.

KEYWORDS: market failures, information disclosure, market, technological products, consumers.

\section{INTRODUCCIÓN}

Los regímenes de divulgación han sido adoptados en una amplia gama de contextos de consumo. La Ley de Valores de 1933, Ley de la bolsa de Valores de 1934, la Ley de Sociedades de Inversión de 1940, y la Ley Sarbanes-Oxley fueron promulgadas para asegurar que los inversionistas estuvieran provistos con información financiera oportuna sobre los valores ofertados. En los mercados de crédito al consumidor, la Ley de Veracidad de Crédito requiere de manera obligatoria y estandarizada la divulgación de los términos y el costo de los prestamos. La verdad en la Ley de Ahorro requiere mandatos claros y uniformes a la divulgación de las tasas de interés y cargos relacionados con las cuentas de depósito para fomentar la competencia y mejorar la toma de decisiones. La ley de garantía Magnuson-Moss requiere que los vendedores de productos de consumo escriban usando un lenguaje claro en forma estandarizada. El etiquetado nutricional y la Ley de Educación exigen que los productores de alimentos detallen el contenido de nutrientes en las etiquetas de sus productos. Muchos otros ejemplos podrían darse.

Un ejemplo reciente, particularmente interesante y uno cercano a la investigación de este artículo, es el Instituto de Leyes Americano, recientemente aprobaron los principios de la Ley de Contratos de "software". Los principios buscan hacer contratos de "software" más accesibles a los consumidores. Específicamente, § 2.02 ofrece salvaguardias para transacciones de mercado masivo, operaciones minoristas dirigiendo una serie de mejoras prácticas del vendedor con respecto a la revelación que, si se aplicaran, aseguren el cumplimiento de condiciones del vendedor. La disposición exige que tanto en línea y proveedores de "software" físico para publicar los términos de sus contratos de licencia estos deben ser de manera "razonablemente 
accesibles" en su sitio web ${ }^{366}$. Esto, por definición, es eliminar el uso de "pagar ahora, las condiciones más tarde" (PNTL) en contratos, que son contratos que los compradores pueden acceder solo después de la compra. Los principios piden que el aviso sea visible, y los términos se encuentren disponibles a través de un hipervínculo antes de comprar "a fin que el cesionario no pueda dejar de ver el anuncio." Los principios también requieren que los vendedores que venden su "software" a través de su propia página web corporativa para hacer clic en "Acepto", junto a un cuadro de desplazamiento que contiene el texto de la licencia.

Si son eficaces, el aumento de la divulgación es una atractiva manera de luchar contra los problemas relacionados con la información imperfecta. Puede ser más barato; es no intrusiva y por tanto no representa un riesgo de mal informada la regulación directa de términos y exigir el cumplimiento de las normas; conserva la elección del consumidor; y, alienta a los vendedores a competir sobre la base de la información divulgada. Como argumenta Beales et al [1981] y [1973] Whitford, la divulgación ataca la causa del problema en contraposición a sus síntomas.

La teoría de regulación basada en la revelación es también elegante. Al reducir los costes de búsqueda, la revelación facilita la comparación de compras y aumenta el número de compradores informados. En principio, un régimen de transparencia puede ser beneficioso incluso si no afecta al comportamiento de la mayoría de los consumidores; puede ser eficaz incluso si la mayoría de los consumidores no leen o tienden a la de comparación basada en lo que leen. Como Schwartz y Wilde [1979] articulan, lo que se requiere es que estos regímenes aumenten el número de consumidores informados a una masa crítica de lo que los autores llaman una "minoría informada." Si esta masa crítica de compradores de comparación existe, divulgación será suficientemente eficaz en mercados competitivos, porque los vendedores tendrán un incentivo para satisfacer informando a los compradores. El no informado no lector consigue un viaje gratis a términos estándar aceptables.

El argumento requiere que los consumidores tengan preferencias homogéneas respecto a términos y que los vendedores son incapaces de discriminar entre los consumidores informados y desinformados.

366 Cabe señalar que incluso los términos que cumplan con el § 2.02(c) (1) estarían sujetos a un reclamo donde los términos son inmorales o en contra de la política pública. 
La minoría informada argumentó que ha sido utilizada ampliamente para resistir la no revelación de la intervención. Pueden encontrarse ejemplos en Spence [1977] y Salop y Stiglitz [1977]. Aúnque los críticos han expresado dudas acerca de la capacidad de divulgación regímenes para generar aumentos significativos en el número de consumidores informados. Goldberg Ben-Shahar [1974], [2009], y Ben-Shahar y Schneider [2010] han argumentado que los consumidores podrían ignorar la impresión, independientemente de cómo sea accesible. Un estudio de especial relevancia aquí es Bakos et al [2011], que encontró que aproximadamente uno de cada mil compradores de "software" online voluntariamente lee el EULA (en el marco de las actuales prácticas de divulgación). O quizás no entienden que cuando lo leen, como Whitford [1973] ha sugerido.

La Economía del comportamiento también nos ha enseñado que los consumidores confían en la heurística para hacer frente a complejos en la toma de decisiones cotidianas. De hecho, varios estudios, incluyendo Bar-Gill [2004] [2008], Gabaix y Laibson [2006], y Della Vigna y Malmandier [2008] sugieren que algunos mercados pueden no estar funcionando de manera óptima debido a diversos tipos de racionalidad. Por ejemplo, Korobkin [2003] explica cómo los consumidores tienden a depender de unos pocos atributos de productos sobresalientes en sus decisiones de compra. Algunas revelaciones que ponen de relieve las pocas, pero más importantes características del producto o servicio podría mejorar la toma de decisiones. Pero, como Issacharoff [2011] NOTAS, no está claro que solo ofrecer más información será útil.

Las apuestas son altas, tanto para los consumidores como para los vendedores. Si las críticas son correctas y los lectores de contrato permanecen inalterables por mayor acceso al contrato, que ordene el aumento de la divulgación sería ineficaz e incluso potencialmente dañino. En primer lugar, los consumidores seguirían tomando decisiones de compra sin considerar plenamente los términos. Segundo, como Ben-Shahar y Hillman [2010] [2006] argumentan, los tribunales podrían creer erróneamente que los vendedores usan un término específico para hacer el producto eficiente, mecanismos de mercado y ser más indulgentes en términos de policía abusiva. En tercer lugar, en el contexto de "software", se requiere la divulgación en forma de "clickwraps" la cual será costosa para los vendedores si los pasos adicionales en el proceso de "checkout" el resultado de compradores 
ansiosos de perderán su paciencia y abandonaran la transacción ${ }^{367}$. Estas recomendaciones podrían generar importantes costosos y cambios en las prácticas de divulgación de información del vendedor del "software" actual, ya que aproximadamente la mitad de los contratos de "software" vendido "online" son actualmente o PNTLs "browsewraps" 368 . También sería costoso para los compradores, que se verían obligados a gastar tiempo manifestando su consentimiento haciendo clic en términos que racionalmente, no tienen ningún interés en revisar.

Al final del día, sin embargo, ninguna cantidad de teorías o debate puede resolver si la revelación de los regímenes es capaz de informar a los consumidores y modelar su comportamiento. Estas son cuestiones puramente empíricas.

\section{1. ¿HA SIDO EFICAZ EL REGLAMENTO DE DIVULGACIÓN? EVIDENCIA PREVIA}

Antes prueba de evidencia empírica sobre la eficacia de los regímenes de divulgación se puede decir que es fragmentada y mixta. Choi et al [2008] se encontró con que los sujetos que están obligados a elegir entre cuatro índices S\&P 500 fondos no suelen elegir aquellos con tarifas más bajas, incluso aúnque esta sería segura para llevar a cabo una mejor base de honorarios netos. Este comportamiento persiste incluso después que los sujetos recibieron folletos que incluyen hojas de resumen de Canon más transparente. En otro conjunto de experimentos por Beshears et al [2008], acortado por SEC revelaciones fueron mostradas solo modestas mejoras en la selección de cartera. Otro estudio realizado por Agarwal et al [2009] se encontró que el joven adulto.

Y relativamente mayores prestatarios más pobres hacen opciones financieras, sugiriendo límites a la capacidad de cualquier régimen de transparencia para mejorar la toma de decisiones.

En el mercado minorista de alimentos, Jin y Leslie [2003] se evidenció que la introducción de la calidad de la higiene estandarizada por

367 Mann y Siebeneicher [2008] encuentran que los minoristas no pueden hallar el valor extra durante el proceso de verificación necesario para obtener su consentimiento legalmente exigible.

368 Este número proviene de un estudio empírico sobre el anterior EULA por Marotta-Wurgler [2009]. 
tarjetas son necesarias para ser mostradas en el restaurante "Ventanas" en Los Ángeles, fue demostrado que el restaurante causó un grado de puntuaciones de inspección para aumentar la sensibilidad de la demanda de los consumidores al aumentar las puntuaciones de higiene, y las hospitalizaciones debidas a enfermedades transmitidas por alimentos Asimismo, Bollinger et al [2010] se comprobó que la introducción obligatoria de contabilizaciones de calorías en cadenas de restaurantes en la Ciudad de Nueva York disminuyó el consumo de calorías en el Starbucks por un modesto 6 por ciento. Un estudio del efecto de la nutrición y la Ley de Educación de etiquetado en el aderezo de ensalada mercado por Mathios [2000] se determinó una reducción estadísticamente significativa en las ventas en aderezos con mayor contenido de grasa después de la aprobación de la ley. Naturalmente, si los consumidores no saben leer o entender las etiquetas, tales regímenes serán menos eficaces. En ese sentido, Lacko y Pappallardo [2007] hallaron que la mejora de la revelación podría aumentar la comprensión de las características complejas de los prestamos.

Algunos han sugerido la divulgación de regímenes son probablemente efectivas solo cuando la revelación se convierte en "incrustada" en la toma de decisiones cotidianas y corrige una percepción errónea del consumidor. Por ejemplo, Bertrand y Morse [2010] evidenciaron que los regímenes de divulgación para prestamos son más eficaces si se toma en consideración los prestatarios sesgos cognitivos.

Divulgación podría por lo tanto ser utilizada como una herramienta de "sesgo" de los individuos o, por lo menos, aquellos que respondan a la información, que en este caso fue solo el diez por ciento de los prestatarios.

\section{OTROS MECANISMOS QUE AYUDAN A QUE LOS MERCA- DOS FUNCIONEN}

Aúnque este artículo se centra en los regímenes de divulgación, es importante señalar que existen otros mecanismos de mercado que pueden inducir a los vendedores sobre la competencia y los términos del contrato, incluso si algunos consumidores los leen. Por ejemplo, como Baird [2006] y otros han señalado, los vendedores afectados por reputación podrían tener un incentivo para ofrecer mejores condiciones o calidad del producto. Jin y Leslie [2009] encontró que la cadena de restaurantes que tienen mayor higiene son decenas de restauran- 
tes independientes, quizás debido a las posibles consecuencias para la reputación. Los vendedores también pueden ser sancionados por la amenaza de litigio, como determinaron Issacharoff y Delaney [2006].

Alternativamente, los vendedores pueden tener un incentivo para divulgar información voluntariamente y publicidad para obtener una ventaja competitiva. Como mencionaron Milgrom [2008] y otros, esto funciona si los compradores son lo suficientemente sofisticados y atentos. Por ejemplo, Mannering y Winston [1995] encontró que la adopción del consumidor de vehículos equipados con "airbags" en el decenio de 1990 parece haber sido facilitado por la cobertura de los medios de comunicación, así como los amigos contaron sus experiencias con airbags. Asimismo, Scott-Morton et al [2001] se comprobó que los consumidores que utilizan Internet como servicio de referencia a la hora de comprar un coche, en promedio pagan menos de $\$ 450$; esta Economía se debe presumiblemente a la información puesta a disposición por el servicio de referencias.

Cuando los compradores son ingenuos y los atributos del producto no son tan agudos como precio o no son denunciados por los medios de comunicación, sin embargo, es posible que estos sigan imperfectamente informados. Este parecería ser la plausible caracterización de muchos, si no la mayoría de los ajustes de contrato de forma estándar. Si es así, se explicaría por qué tantos regímenes de divulgación se han propuesto.

\section{3. ¿LA DIVULGACIÓN DE CONTRATO ES IMPORTANTE? UNA APROXIMACIÓN EMPÍRICA}

Dada la variedad de información existente y propuesta sobre la divulgación de contrato y los efectos potenciales sobre los vendedores, es importante ampliar el conjunto de pruebas sobre si una política de aumento de la divulgación de contratos de forma estándar puede informar eficazmente a los consumidores y crear una minoría informada de compradores capaces de disciplinar a los vendedores. De hecho, eso parecería ser un requisito previo para la regulación.

Mi enfoque es estudiar el comportamiento de aquellos que compran productos de software en línea. Rastreo el comportamiento de compra de los visitantes de Internet a 81 minoristas de software que venden sus productos en su sitio web corporativo y que hicieron sus licencias de "software" disponible en algún lugar de su sitio. Examino la tarifa 
a la cual los compradores eligen para informarse sobre las licencias de "software" que posee el software destacado. Particularmente, estudio si los consumidores son más propensos a acceder a las licencias de "software" que están prominentemente visualizadas.

El mercado de productos de software en línea es un entorno particularmente bueno para examinar la eficacia potencial de las políticas de divulgación de contratos. En primer lugar, las licencias de "software" incluyen importantes características que no están relacionadas con el precio, como los Derechos y restricciones sobre cómo el producto de "software" puede y no puede utilizarse. Los términos de las licencias de "software" siguen estando en el centro de varias disputas legales $^{369}$. Segundo, como se mencionó anteriormente, el Instituto de Derecho Americano ha aprobado recientemente los nuevos Principios de Derecho sobre los Contratos de "Software". Estos se redactaron bajo la suposición que el mercado masivo de "software" no funciona bien con respectos a los términos contractuales. Esta suposición no es irrazonable, dadas las pruebas que actualmente no existe una minoría informada en el mercado de "software" en línea. Saber si el aumento de la divulgación de contrato es realmente capaz de crear una minoría informada de compradores de "software" es obviamente crucial para evaluar la conveniencia de este enfoque. Por último, una serie de debates sobre la reforma legal y el papel de regulación de la divulgación en los contratos tipo que generalmente se centran en los contratos electrónicos y particularmente en los contratos de "software".

Para estimar si el aumento de la divulgación de contratos está asociado con un mayor número de lectores, empiezo clasificando los visitantes de los sitios web de la empresa de mi muestra en aquellos que tienen la intención de comprar y los que visitan por otros motivos. Por ejemplos, algunos visitantes pueden visitar un minorista de "software" para averiguar cómo solucionar un problema en una copia que ya tienen, o para ver anuncios. Utilizo el enfoque de Bakos, Marotta-Wurgler, y Trossen (2011) para distinguir a los adquirientes de los no adquirientes. También distingo los adquirientes que compran un producto de aquellos que no lo hacen y los clasifico como compradores potenciales o no potenciales. Utilizo la iniciación

369 Algunos ejemplos pueden ser encontrados en Altera Corp v. Clear Logic, Inc. 424 F.3d 1079 (9no Cir. 2005); Davidson \& Assocs. v. Jung, 422 F.3d 630 (88vo Cir, 2005); Davidson \& Assocs. v. Internet Gateway, 344 F. Supp. 2d 1164, 1178 (D. Mo. 2004); M. A. Morteson Co. V. Timberline Software Corp., 998 P.2d 305 (Wash. 2000) 
de un proceso de pago seguro para identificar a los compradores potenciales. Finalmente, mido el número de visitantes - adquirientes y compradores potenciales - que leen las licencias de contrato. Es decir, estimo el número de lectores y no lectores entre los visitantes clasificados como adquirientes y compradores potenciales. Defino a los lectores como aquellos que acceden a una página de licencia de "software" durante más de un segundo. Esto da un límite superior al número de personas que efectivamente leen contratos, ya que algunos pueden hacer clic en ellos accidentalmente, y algunos no pasan el tiempo suficiente en ellos como para haber entendido sus términos.

\subsection{Datos y Construcción de Muestras}

El conjunto de datos contiene las rutas de navegación web de 92.411 hogares de los Estados Unidos para enero de 2007 y fue introducido en Bakos, Marotta- Wurgler, and Trossen (2011). Estos datos fueron recogidos por una importante compañía de investigación en línea que realiza un seguimiento del comportamiento de navegación por Internet de un panel representativo de hogares de EE.UU, que han acordado instalar en sus computadores un programa que rastrea la URL de cada página visitada durante sus sesiones de Internet.

El panel de hogares fue seleccionado para ser representativo de la población de hogares Estadounidenses con acceso a Internet. A cada hogar se le asigna un identificador anónimo único, que se utiliza para rastrear su actividad de navegación web y para clasificarla en "sesiones". La información captada por el "software" incluye la URL de cada página visitada, el tiempo dedicado a la página, si esa página estaba dentro de una conexión segura (es decir, encriptada), el servidor web que entrega la página web y un identificador único para la empresa o la división propietaria de ese servidor web. La compañía también recoge información demográfica detallada entre los hogares.

Construyo la muestra seleccionando las visitas de los usuarios a los minoristas que venden productos de "software" en su sitio web corporativo y que hacen que sus contratos estén disponibles en algún lugar de su sitio, antes o durante el proceso de pago. Uso la clasificación del proveedor de datos de los mercados para identificar las visitas solo a las empresas de "software". Excluyo a los proveedores que ofrecen sus productos de forma gratuita (por ejemplo, proveedores de "software" gratuito), los proveedores que no venden su producto a través de su 
sitio web corporativo, los proveedores de "software" de igual a igual y las empresas de alojamiento web. Incluyo solamente a las compañías con por lo menos cincuenta visitantes únicos que vieron por lo menos dos páginas. Mi interés está en los usuarios con intención o potencial intención de compra, y los usuarios que ven una sola página son menos propensos a tener tal intención. Un total de ochenta y un empresas satisfacían las condiciones anteriores.

Para cada una de estas empresas obtuve manualmente los URL de todas las licencias de "software" disponibles en el sitio web de la empresa, independientemente de dónde se encuentren. Algunas compañías ponen sus licencias de "software" a disposición de los visitantes antes de comprarlas publicándolas en alguna parte de su sitio web. Otros las presentan durante el proceso de pago y requieren que los compradores hagan clic en "estoy de acuerdo", ya sea junto a un hipervínculo que dirige al usuario a los términos del contrato o debajo de un cuadro de texto que contiene los términos de licencia. Todas estas empresas forman parte de la muestra, aúnque, como se explica a continuación, el subconjunto que presenta términos en un cuadro de texto se analiza mejor por separado.

\subsection{Características de la Empresa y de los Productos}

Recopilé información de la compañía y del producto que podría afectar la propensión de un comprador a estar informado sobre los términos de la licencia de "software". Obtengo datos sobre los ingresos anuales de cada empresa (en 2009), año de incorporación y Estado público o privado. Estos datos proceden de fuentes públicas o de comunicaciones directas con las empresas.

Los datos a nivel de empresa se describen en la Tabla 1. El ingreso promedio de las 81 compañías de muestra es de \$ 1,52 mil millones, cifra impulsada por unas pocas grandes empresas; el ingreso medio es de solo seis millones de dólares. La edad media de estas empresas, medidas como 2011 menos el año de incorporación, es de 17,7 años. Aproximadamente una cuarta parte de las empresas se comercializan públicamente.

Recojo datos relacionados con productos para el producto insignia de cada empresa. Para empresas con muchos productos, selecciono el que es más popular o que representa la mayor parte de las ventas. Si tal información no está disponible, selecciono el producto más 
prominente ofrecido por el vendedor. Registro el precio del producto ofrecido y recojo la información sobre el precio medio de todos los otros productos ofrecidos por cada vendedor, para tener una idea de lo representativo que es el producto destacado. Observo si el producto destacado es un acuerdo de licencia única o multilicencia y si está dirigido a desarrolladores de "software", ya que esto afecta el precio. También observo si los vendedores ofrecen una versión de prueba del producto, y si el producto está dirigido a usuarios comerciales o al público en general. Por último, clasifico cada producto en una de las 150 categorías de productos de "software", por ejemplo, gráficos u hojas de cálculo, en función de las clasificaciones que utiliza Amazon.com. 


\begin{tabular}{|c|c|c|c|c|c|}
\hline \multicolumn{6}{|l|}{ Tabla 1} \\
\hline \multicolumn{6}{|c|}{ Muestras de empresas de software y sus productos } \\
\hline & $\begin{array}{l}\text { Núme- } \\
\text { ro }\end{array}$ & Media & $\begin{array}{l}\text { Mínimo } \\
\text { (D.D) }\end{array}$ & Mediana & Máxima \\
\hline \multicolumn{6}{|c|}{ Panel A: Características de la Empresa } \\
\hline $\begin{array}{l}\text { Ingresos } \\
\text { (\$millones) }\end{array}$ & 81 & $\begin{array}{c}1.520 \\
(6,850)\end{array}$ & 0.1 & 6 & 51.100 \\
\hline $\begin{array}{l}\text { Tiempo } \\
\text { (años) }\end{array}$ & 81 & $17.7(9.98)$ & 5 & 16 & 58 \\
\hline $\begin{array}{l}\text { Compañía } \\
\text { Pública }\end{array}$ & 81 & $0.27(0.45)$ & 0 & 0 & 1 \\
\hline \multicolumn{6}{|c|}{ Panel B: Características del Producto } \\
\hline $\begin{array}{l}\text { Producto del } \\
\text { Consumidor }\end{array}$ & 81 & $0.68(0.47)$ & 0 & 1 & 1 \\
\hline Precio $(\$)$ & 81 & $394(1,025)$ & 10 & 58 & 5.290 \\
\hline Precio Medio & 81 & $352(1,025)$ & 1 & 49 & 5.000 \\
\hline $\begin{array}{l}\text { Juicio } \\
\text { (producto } \\
\text { destacado) }\end{array}$ & 81 & $0.84(0.37)$ & 0 & 1 & 1 \\
\hline $\begin{array}{l}\text { Juicio } \\
\text { (mayoría del } \\
\text { producto) }\end{array}$ & 81 & $0.78(0.42)$ & 0 & 1 & 1 \\
\hline
\end{tabular}

La tabla 1 también proporciona estadísticas resumidas sobre los productos estrella de cada empresa. El sesenta y ocho por ciento está dirigido a los miembros del público en general. El precio promedio de los productos destacados es de \$394, y la mediana es de \$ 58. Los productos destacados son representativos de los productos de la empresa, al menos en lo que respecta al precio, dado que los precios promedio de todos los productos de software están cerca de estos. Esto es útil 
mientras examino el acceso a todos los productos para los que hay una licencia de software publicada. Finalmente, el ochenta y cuatro por ciento de los sitios de la muestra ofrecen una versión de prueba del producto ofrecido o del producto en la muestra, y setenta y ocho por ciento ofrecen versiones de prueba para la mayoría de sus productos.

\subsection{Divulgación de Contrato}

Uno de los dos objetivos empíricos de este artículo es medir si los contratos más accesibles son más probables de ser leídos (el otro es, dada la lectura, si lo que está en el contrato afecta a las decisiones de compra). Por lo tanto, recojo todas las URL de las licencias de "software" que están disponibles en el sitio web de una empresa. Como se señaló anteriormente, muchas empresas venden un solo producto y, por lo tanto, solo ponen en línea la licencia de "software" que rige el uso de ese producto. Otras firmas venden muchos productos que están regidos por una sola licencia de "software" publicada en su sitio web, y otros publican diferentes licencias de software para diferentes productos. Registro cada licencia de "software" publicada. Esto me permite examinar las visitas a todos los productos con una licencia de "software" asociada en una empresa dada, no solo los productos estrella. Existen 240 URL únicas que corresponden a licencias de "software" para mis empresas de muestra.

Mido la accesibilidad del contrato con el número de clics al ratón que toma para tener acceso a la licencia de software desde el camino más natural para comprar. Definir el "camino natural para comprar" puede sonar complicado y subjetivo, pero en realidad es un proceso sencillo, ya que la mayoría de las empresas tratan de hacer lo más fácil posible para que los consumidores puedan navegar a través de sus sitios y comprar sus productos.

Por ejemplo, considere a un comprador que desea adquirir un producto de software antivirus de un determinado proveedor. El comprador seleccionará el producto y procederá a la comprobación. Imagine que antes que se le permita ingresar su información de tarjeta de crédito, el comprador debe aceptar la licencia de "software" del producto haciendo clic en "Estoy de acuerdo" debajo de una caja desplazada que contiene los términos estándar, este es un contrato de clic. Debido a que la licencia de "software" está directamente en el camino de clic más natural para los compradores y se requiere verlo. Si el vendedor hubiera pedido 
a los compradores que hicieran clic en "Estoy de acuerdo", pero los invite a acceder a la licencia de "software" haciendo clic una vez más en un hipervínculo adyacente, además, registro el puntaje de acceso como 0,5 . Esto es porque, aúnque el comprador debe reconocer activamente la existencia de un contrato, y por lo que en ese sentido es muy fácil de encontrar, también debe hacer clic una vez para ver sus términos. Estos dos tipos de presentaciones de contrato fuerzan a los compradores de la licencia de "software" a reconocerla.

La distribución de la accesibilidad del contrato por parte de la empresa se presenta en la Tabla 2. A partir de la fecha del proceso de recolección de datos, el 3,70\% de los vendedores colocan cajas de texto con los términos sobre el botón "Estoy de acuerdo" -la máxima divulgación posible- y el 27,16\% de los vendedores los presentan en hipervínculos cercanos que deben hacer clic para ver los términos.

El resto de las empresas de la muestra hacen sus contratos disponibles en su sitio web, pero requieren que los compradores hagan un esfuerzo extra para encontrarlos. Por ejemplo, Symantec presenta toda su licencia de productos a un mínimo de dos clics de distancia de la ruta de compra más obvia. Al momento de escribir este artículo, un enlace en la parte inferior de la página principal, titulado "acuerdos de licencia", proporciona enlaces a las licencias de "software" de los productos de Symantec. Por lo que toma a un comprador un clic de la página principal para acceder a la lista de las licencias de "software", y el segundo clic para ver realmente la licencia de "software" del producto deseado, por una distancia total de dos clics. Alrededor del cuarenta y dos por ciento de los vendedores colocan su licencia a un solo clic; en todas las empresas que no son del carácter obligatorio el "Estoy de acuerdo" o el carácter de una ruta de navegación, el número medio de clics necesarios para ver la licencia de "software" es de 1.66. Uno debe estar determinado, de hecho, a encontrar algunos de estos contratos, ya que los enlaces no siempre están claramente etiquetados, y el texto puede ser muy pequeño (quizás intencionalmente). A priori, la tabla sugiere que la preocupación del regulador sobre las prácticas actuales de divulgación no es injustificada. 


\begin{tabular}{|c|c|c|}
\hline Tabla 2 & & \\
\hline ¿Qué tan bien se revelan & s licencias de "software & \\
\hline $\begin{array}{l}\text { Revelación de la } \\
\text { licencia de "software" } \\
\text { (número de clics de la } \\
\text { ruta de compra) }\end{array}$ & $\begin{array}{l}\text { Número de las } \\
\text { licencias de software }\end{array}$ & Porcentaje \\
\hline 0 (ruta de navegación) & 3 & 3,7 \\
\hline $\begin{array}{l}0.5 \text { (ruta de } \\
\text { navegación) }\end{array}$ & 22 & 27,16 \\
\hline 1 & 34 & 41,98 \\
\hline 2 & 12 & 14,81 \\
\hline 3 & 7 & 8,64 \\
\hline 4 & 2 & 2,47 \\
\hline 5 & 0 & 0 \\
\hline 6 & 1 & 1,23 \\
\hline Total & 81 & 100 \\
\hline
\end{tabular}

\subsection{Compradores y Compras}

Estoy interesado en observar el comportamiento de los visitantes que tienen la intención de comprar. El proveedor de datos informa toda la actividad de navegación de Internet de mis visitantes, por lo que no puedo saber con seguridad qué fracción de los visitantes de los minoristas de muestra son verdaderos compradores o tienen otras motivaciones. Empezaré restringiendo mi análisis a los visitantes con intenciones potenciales de compra excluyendo visitas que no tengan acceso a servidores dedicados a comprar o adquirir (por ejemplo, servidores que tratan de promociones). A continuación, sigo el enfoque en Bakos, Marotta-Wurgler, and Trosen (2011) para identificar las visitas orientadas a las compras con mayor precisión. Esto tiene alguna explicación. 
Defino la visita de usuario como todas las vistas de páginas (accesos URLS) desde el sitio web de una empresa dentro de una única sesión de usuario. Un enfoque ampliamente utilizado en la secuencia de clics es identificar a los compradores centrándose en la intensidad de una visita de la empresa. Los usuarios con la intención de considerar una compra -los llamo adquirientes- tienen más probabilidades de ver varias páginas en el lado minorista del sitio web de la compañía. Esta observación me ayuda a separar el navegador casual de los compradores adquirientes.

En concreto, he utilizado dos definiciones de visitas de compras para estimar si el usuario estaba de compras. La definición más amplia de visitas de compras es aquella en la que al menos cinco páginas se accedieron en el sitio web de una compañía dada. Esta definición debería excluir muchos de los navegadores casuales. Una definición mucho más restrictiva de una visita de compras es aquella en la que el usuario ha seleccionado un producto e iniciado una revisión o pago en una sesión determinada. Identifico a estos visitantes mediante el inicio del proceso de pago. Esta definición es excesivamente restrictiva, ya que excluye a muchos compradores que no compran en última instancia. Dada una definición demasiado permisiva de visita de compras y una definición demasiado restrictiva, puedo establecer límites superiores e inferiores sobre las cantidades de interés. En este caso, como los intervalos acotados resultan ser muy ajustados, la definición precisa de comprador es inmaterial.

Una vez que identifico a los compradores, defino una visita de empresa como un período de navegación web separado por al menos treinta minutos de inactividad. Un usuario puede, por lo tanto, tener varias visitas a una empresa dada en un día, una semana o un mes. El proveedor de datos utiliza esta definición, al igual que varios artículos de la literatura, como Moe y Fader (2004) y Bucklin y Sismeiro (2003) ${ }^{370}$. Me refiero a todas las vistas de página desde el sitio web de una sola compañía dentro

370 Aúnque es útil, esta definición puede ser demasiado estrecha, ya que los visitantes en línea pueden considerar su decisión de compra con el tiempo y visitar una empresa en varias ocasiones, durante el lapso de varios días, antes de completar una compra. Johnson et. al (2004) demuestra que las visitas repetidas a una empresa dentro de un mes típicamente corresponden al mismo ciclo de compras. Por lo tanto, se trata de una definición alternativa de la visita de la empresa mediante la agregación del número de visitas a una sola empresa en un mes determinado. Los resultados de este enfoque fueron cualitativa y cuantitativamente similares y están disponibles a petición. 
de una sola sesión de usuario como una visita de la empresa por parte del usuario. El número de visitantes únicos en la muestra bajo la definición más amplia de visitas de compras, que es de al menos cinco páginas vistas en el sitio web de esa compañía, es 35000.

El proveedor de datos también reúne datos demográficos sobre compradores y hogares que pueden afectar su probabilidad de ser informados sobre los términos de la licencia. Utilizo datos sobre la edad y el género del jefe del hogar, el ingreso del hogar, el tamaño del hogar y la presencia de niños. Debido a que la muestra se construye para ser representativa de los usuarios de Internet, y estos datos son simplemente variables de control en el análisis, me abstengo de informar sus efectos numéricos para ahorrar espacio, pero están disponibles a petición.

\section{4. ¿̇SON LOS CONTRATOS MÁS ACCESIBLES MÁS PROBA- BLES DE SER LEÍDOS?}

Aquí yo pruebo si los contratos que requieren menos clics extras son más probables de ser leídos, o al menos vistos. Yo me enfoco en la frecuencia con la que los compradores acceden al URL de una licencia de software, y si esta frecuencia incrementa de tal manera que los contractos son hechos fáciles de encontrar, yo computo estadística descriptiva en visitas de compañías y accesos de licencia por accesibilidad de contrato, bajo cada dos definiciones de visita de compras. Yo logro esto con una regresión simple para estimar el efecto de la accesibilidad de contratos en acceso a contratos mientras se toma en cuenta una gama de variables de control.

\subsection{La relación entre lectura de contrato y revelación}

Para esta porción del análisis, yo me enfoco en visitas a compañías donde el acceso EULA es posible, pero es opcional. En otras palabras, yo excluyo las compañías con una calificación de acceso EULA de cero, porque todos los compradores que empiezan el proceso de compra están presentados con el texto de EULA. Para ellos la fracción que voluntariamente "lee" no puede ser determinada.

7) Aunque útil, esta definición puede ser muy estrecha, los visitantes en línea pueden considerar sus decisiones de compra a través del tiempo, y visitar una compañía en múltiples ocasiones, en el 
lapso de varios días antes de completar una compra. Johnson et al. (2004) muestra que las visitas repetidas a una empresa dentro de un mes normalmente corresponden al mismo ciclo de compras. Por lo tanto, traté de una definición alternativa de visita de compañía agregando el número de visitas a una única compañía en un mes determinado. Los resultados de este enfoque fueron cualitativa y cuantitativamente similares y disponibles bajo petición.

8) La mayoría de las firmas tienen la misma calificación de accesibilidad para todos sus productos EULA. Muy pocas firmas hacen sus EULAs disponibles en múltiples ocasiones. En esos casos, yo registro las puntuaciones más bajas (i.e. más accesibles) ¿̇Importa la divulgación de contrato?

Los resultados esenciales de esta investigación se encuentran en la Tabla 3. El panel superior mira las visitas ininterrumpidas de aquellos que hicieron clic en al menos cinco páginas durante una visita de la empresa. Comienza desde la parte inferior de este panel, y busca hasta qué punto los compradores accedieron al EULA de la empresa que lo hizo más difícil de encontrar, a seis clics de distancia. (De la Tabla 2, sabemos que sólo hay una compañía de este tipo.) De 235 visitas de compras a esta empresa, incluyendo visitas repetidas, el EULA no fue accedida ni siquiera una vez. Las 1.160 visitas de compras a sitios web donde el EULA estaba a cuatro clics de distancia de la ruta de compra tampoco presenciaron un solo clic en un EULA. Parece plausible conjeturar que al menos algunos compradores pudieron haber Estado interesados en revisar estos contratos, pero simplemente no los pudieron encontrar.

La imagen no cambia mucho a medida que aumenta la divulgación. De las 49,079 visitas de compras a las empresas que localizaron sus EULAs a tres clics de la ruta de compra, solo trece vieron a EULA accedida, una tasa de tres accesos EULA por cada diez mil visitas de compras (es decir, accesos EULA potenciales). Cuando la revelación se mejora para estar un clic más cerca de la ruta de compra, para un total de dos clics adicionales requeridos, la tasa de lectores en realidad cae ligeramente, a alrededor de uno de cada nueve mil. Cuando está un clic más cerca aún, ahora solo requiere un solo clic en un hipervínculo que está realmente en una página de ruta de compra, la tasa de lectura aumenta, pero sigue siendo pequeña: es solo alrededor de uno de cada trescientos compradores. 
Hubo 4.513 visitas de compras a las empresas que hacen su contrato disponible a través de un clickwrap, incluyendo visitas repetidas. Los compradores accedieron voluntariamente al EULA de estas compañías sólo siete veces. En otras palabras, sólo una fracción del uno por ciento de las visitas de los compradores incluyó un clic de EULA. Note que, con estos clickwraps, el EULA es tan bien divulgado como posiblemente puede ser corto de ser impreso en la pantalla a lo largo de la ruta de compra, que como vimos anteriormente es un método de divulgación elegido por sólo tres de las 81 firmas.

Muchos de los que hicieron clic en el EULA probablemente no lo han leído y comprendido. Podemos ver esto en el tiempo invertido en la URL de la licencia. La media del tiempo para que un "lector" pase en esa URL es entre treinta y sesenta segundos (el tiempo promedio en EULA oscila entre cuarenta y ciento cincuenta segundos). Bailey y Bailey [1999] encontraron que el EULA promedio es de aproximadamente 2.300 palabras de largo, y la velocidad de lectura humana promedio es menos de trescientas palabras por minuto. La velocidad de lectura es sin duda más baja para el juez legal. Por lo tanto, las bajas tasas de clics EULA que observamos aún exagera la fracción de compradores que realmente se informa sobre los términos de la licencia.

El segundo panel de la Tabla 3 muestra los resultados para los compradores que fueron todo el camino a la colocación de un producto en un carrito de la compra y el acceso a una página de pago seguro. Muchos de estos son por lo tanto compradores reales, mientras que algunos no completan el proceso de pago. El número de visitas en este grupo es menor que en los dos anteriores, de acuerdo con la evidencia previa de las tasas de conversión en línea alrededor del dos por ciento. El patrón de visitas a los EULAs por accesibilidad también es ligeramente diferente bajo esta definición de una visita de compras. Presumiblemente, este grupo de compradores estaría especialmente interesado en ver los términos que están a punto de gobernar el uso de su potencial compra. Sin embargo, esto no parece ser el caso; casi ninguno de ellos accedió voluntariamente a la licencia. 
Tabla 3

¿Más divulgación induce más lectura de licencia?

\begin{tabular}{|l|l|l|l|l}
\hline $\begin{array}{l}\text { Divulgación } \\
\text { de EULA } \\
\text { (número de } \\
\text { clics de ruta } \\
\text { de compra) }\end{array}$ & $\begin{array}{l}\text { Número de } \\
\text { visitas al } \\
\text { sitio web de } \\
\text { la empresa }\end{array}$ & $\begin{array}{l}\text { Número de } \\
\text { visitas con } \\
\text { EULA hecho } \\
\text { clic }\end{array}$ & $\begin{array}{l}\text { Porcentaje } \\
\text { de visitas } \\
\text { con EULA } \\
\text { hecho clic }\end{array}$ & $\begin{array}{l}\text { Mediana de } \\
\text { la duración } \\
\text { de la vista } \\
\text { EULA } \\
\text { (segundos) }\end{array}$ \\
\hline
\end{tabular}

Panel A: Al menos cinco páginas visitadas durante la visita

\begin{tabular}{|c|c|c|c|c|}
\hline 0.5 & 4513 & 7 & 0.16 & 58 \\
\hline 1 & 8110 & 23 & 0.28 & 27 \\
\hline 2 & 9185 & 1 & 0.01 & 44 \\
\hline 3 & 49079 & 13 & 0.03 & 35 \\
\hline 4 & 1160 & 0 & 0 & - \\
\hline 5 & 0 & - & - & - \\
\hline 6 & 235 & 0 & 0 & - \\
\hline
\end{tabular}

Panel B. Al menos una página de Secure Checkout visitada durante la visita

\begin{tabular}{|c|c|c|c|c|}
\hline 0.5 & 381 & 2 & 0.52 & 372 \\
\hline 1 & 3157 & 4 & 0.13 & 77 \\
\hline 2 & 1111 & 0 & 0 & - \\
\hline 3 & 105 & 0 & 0 & - \\
\hline 4 & 112 & 0 & 0 & - \\
\hline 5 & 0 & - & - & - \\
\hline 6 & 0 & 0 & 0 & - \\
\hline
\end{tabular}


Una vez más, todos los compradores que visitan empresas con una puntuación de acceso de 0,5 EULA son conscientes del EULA porque el proceso de pago requiere que ellos lo acepten explícitamente. Sin embargo, incluso para este grupo de compradores particularmente interesados, solo alrededor de uno de cada doscientos clics el EULA. Parece que una mayor divulgación es esencialmente incapaz de inducir a los compradores a estudiar los términos, incluso cuando tales compradores reciben una notificación muy clara al ser obligados a hacer clic en "Estoy de acuerdo" con dichos términos.

Resumamos los resultados. Es estadísticamente cierto que el aumento de la divulgación aumenta el número de lectores. En las regresiones no registradas (disponibles bajo petición), estos resultados se mantienen después de controlar los controles de productos, empresas y compradores. Las tasas de acceso a EULA tienden a aumentar cuando se ubican más cerca del camino de compra. Sin embargo, esto claramente no es adecuado para llevar, porque el aumento es de una tasa de cero a una tasa de épsilon, incluso para los EULAs más prominentemente divulgados y el grupo de compradores más afectados. En el contexto actual, la divulgación creciente no aumenta, y lo más probable es que no pueda, incrementar el número de lectores de los contratos a una tasa significativa.

9) Los resultados son similares para los modelos de probabilidad lineal, logit y probit.

Ahora vuelvo a los contratos mejor divulgados analizando el tiempo dedicado a aquellos donde los vendedores los presentan en un cuadro de texto junto a una caja que los compradores deben aceptar, lo más comúnmente posible durante el proceso de pago. Anteriormente me referí a estos contratos como tener una puntuación de acceso de cero. Solo puedo medir el tiempo dedicado a estas páginas, ya que todos los compradores que deciden comprar un producto son presentados con el texto de EULA, si quieren verlo o no.

Las compañías que eligen este modo de presentación también pueden requerir que el comprador escriba su nombre, dirección de facturación e información de tarjeta de crédito en la misma página donde aparece el texto de EULA, por lo que el método de evaluar el lector basado en el tiempo dedicado a esa página es algo menor de confianza. 
Note que los números a seguir no se informan en ninguna tabla para ahorrar espacio. En mis datos hay 3.269 visitas a firmas con EULAs con una puntuación de acceso cero, donde visita se define como acceso de al menos cinco páginas. De estos, el 14,4 por ciento visitan la página del EULA, donde pasan un tiempo medio de 68 segundos. La manera de interpretar este resultado es que el 14,4 por ciento de los visitantes comenzó, pero no necesariamente completó, un proceso de pago. Dado que estas empresas a menudo requieren que los compradores ingresen información personal y acepten el EULA en la misma página, probablemente los pasos que proporcionan información representen la mayor parte del tiempo que pasan en esta página. Pero de nuevo, incluso si descartamos el requisito de ingresar información personal, es improbable que haya mucha lectura o comprensión de los términos dado el tiempo que pasamos en la página.

Finalmente, limitando la consideración a las visitas con los compradores que comenzaron un proceso de pago, el número de visitas de la empresa cae a 643. Dado que el EULA se presenta durante el registro o en algún momento durante el proceso de pago, no es sorprendente que 302 de las 643 visitas de la compañía (o 47 por ciento) también tuvieron una visita de EULA. El 53 por ciento de las visitas que no tienen una vista EULA son situaciones en las que los compradores comenzaron un proceso de pago o registro, pero no llegaron lo suficientemente lejos para acceder a la URL de la licencia. El tiempo promedio dedicado a estas páginas, cuando se accede, fue de 146 segundos. La conclusión general sigue siendo válida: no importa cuán prominentemente se divulguen los EULAs, la gran mayoría de los compradores no los leen.

Una posible explicación es que a pesar que este tipo de EULA está visiblemente colocado en una página, a menudo aparece solo al final de la transacción durante el proceso de compra, que se inicia después que un comprador ya ha decidido comprar. Los compradores que en teoría podrían ser parte de la minoría informada, acceder a los términos de EULA como parte de su consideración de un producto para la compra, podría resultar demasiado costoso para seleccionar un producto y comenzar un proceso de pago solo para acceder a la licencia. En ese sentido, una licencia que es uno o incluso dos clics de distancia de la página principal de la empresa podría ser más "accesible", incluso si no es deliberadamente presentado a todos los compradores.

Del mismo modo, una vez que un comprador ha decidido comprar un producto y ha iniciado una sesión de pago sin tener en cuenta los 
términos del EULA, tal vez no se preocupe por el EULA. Esto también podría explicar el poco tiempo dedicado a los EULA presentados durante el proceso de pago.

En cualquier caso, la pequeña fracción de consumidores que acceden a EULAs indica que los compradores perciben un alto costo de encontrar y leer la licencia en relación con los beneficios esperados. Dado que el número de lectores de los contratos no aumenta significativamente con el aumento de la divulgación, el principal costo no radica en localizar y acceder a los EULAs, sino más bien en la lectura y evaluación de los términos del contrato.

Los contratos pueden ser demasiado largos o difíciles de entender, o los consumidores pueden no importarle lo suficiente acerca de los términos para incurrir en el costo de leerlos. Aúnque varios términos implican restricciones de uso, garantías y mantenimiento y soporte, la mayoría de términos son relevantes solo en eventos de baja probabilidad. La falta de lectura sin tener en cuenta la revelación podría ser así una acción perfectamente racional. La conclusión de la política es que es poco probable que la divulgación obligatoria afecte significativamente a la fracción de consumidores que se informan.

Para poner la misma conclusión en términos más teóricos y explorar su implicación para la posible eficiencia de los términos vendedores presentes, tenga en cuenta que Bakos, Marota Wurgler, and Trosen (2011) estimaron la fracción mínima de lectores necesaria para apoyar un equilibrio minoritario informado. En ausencia de asentimiento obligatorio y con suposiciones conservadoras sobre el costo de proporcionar términos de contrato favorables, encuentran que la fracción de compradores que tienen acceso a EULAs es entre uno y dos órdenes de magnitud menos de lo que se requeriría para apoyar a una minoría informada en equilibrio. Mi análisis no ofrece evidencia que el aumento de la accesibilidad o el consentimiento obligatorio por sí mismo podría aumentar el número de lectores lo suficiente como para cambiar esta conclusión. El aumento de la divulgación o el consentimiento obligatorio simplemente no elevaría el número de lectores lo suficiente como para ejercer una presión competitiva sobre los vendedores. 


\section{2. $\dot{i}$ Se pueden informar los consumidores sin leer el contrato?}

Tal vez los consumidores se informen sobre los términos consultando sitios web relevantes, tales como "Consumer Reports", blogs dedicados a exponer a los vendedores con malos términos, o puntos de noticias especializados que podrían discutir el contenido de los términos de los contratos estándar, según Becher y Zarsky [2008]. Bakos, Marotta Wurgler y Trossen [2011] exploras este tema y yo resumo sus resultados. Obtienen una lista de los veinticinco sitios más traficados que probablemente tengan información sobre los términos del EULA. A continuación, revisan las páginas particulares a las que acceden los compradores de cada uno de estos sitios para determinar si buscan información sobre los términos del EULA o la calidad del producto. Ellos encuentran que los compradores accedieron a la información de EULA en sitios de revisión de consumidores en solo tres de 148.552 sesiones con al menos dos páginas visitadas (una definición más inclusiva de visita de compras que la mía). Además, menos de cien compradores accedieron a páginas de noticias con información general sobre productos de software en particular, no necesariamente los productos para los que estaban comprando. Por lo tanto, es poco probable que los compradores estén informados por otras fuentes de Internet. Chari [2010] encuentra que las calificaciones de calidad de software en sitios de revisión populares no están correlacionadas con la calidad del contrato. Pero incluso si algunos compradores consultaran dichos sitios web para informarse, esto no afectaría la conclusión sobre la eficacia de la divulgación por parte de los vendedores.

Otro método de volverse "informado" sin leer es confiar en la reputación de los vendedores al decidir si comprar. De hecho, Bakos, Marotta Wurgler y Trossen [2011] encuentran un apoyo moderado para esta posibilidad; los compradores son ligeramente más propensos a acceder a los EULAs de las empresas más pequeñas que podrían no ser tan bien conocidas por los consumidores. Pero incluso si los vendedores se ven limitados por la reputación, sigue siendo el caso que el mandato de divulgación del contrato no va a cambiar el comportamiento del consumidor. Los vendedores pueden o no ser disciplinados por preocupaciones de reputación, pero no están siendo disciplinados por el mecanismo minoritario informado que el aumento de la divulgación espera crear. 
10) Esto podría ser particularmente cierto para los productos de bajo precio. Sin embargo, incluso cuando restrinjo la muestra a productos que cuestan \$ 500 o más, el número de lectores de EULA sigue siendo bajo. Los productos más caros de la muestra cuestan varios miles de dólares, por lo que es difícil atribuir el número bajo de lectores de EULA al valor del bien para los productos de este rango. También compruebo si los compradores tienen menos probabilidades de leer los EULA de los productos que tienen más probabilidades de ser comprados repetidamente, como el procesamiento de textos. No encuentro ninguna relación entre la naturaleza del uso del software y la propensión de los usuarios a acceder a los EULAs.

\section{3. ¿Se preocupan los consumidores por lo que leen?}

Esta sección del artículo, explora una segunda condición necesaria para una efectiva regulación de divulgación: para aquellas personas quienes lean contratos de servicio en este artículo. Esta investigación también tiene implicaciones para un diseño de cualquier propuesta de regulación, para aquellos consumidores que no entienden términos o leyes de una manera que maximice sus intereses propios, la manera en la cual la información convertida necesita ser cambiada. Por ejemplo, los contratos pueden ser simplificado, o escritos en un lenguaje más planificado, o que tengan palabras claves de referencia alta. Yo examino si los consumidores que leen contratos de compradores son consumidores más susceptibles a seleccionar un producto.

\subsection{Una Mirada más Cercana al Contenido del Contrato}

Refiriéndose al trabajo anterior, yo mido el sesgo de un contrato basado en como este trata veintitrés términos comunes que asignan Derechos y riesgos entre compradores y vendedores. Como se indica en la Tabla 4, esto incluye términos relacionados con la aceptación de la licencia, alcance, restricciones de transferencia, garantías y declinaciones de garantías, limitaciones de responsabilidad, mantenimiento y soporte, y solución de conflictos. Yo mido el relativo entre consumidor y amabilidad de estos términos en contra la relevancia preestablecida en las reglas, es decir, el artículo 2 del Código de Comercio Uniforme. Estas reglas preestablecidas gobiernan las relaciones de las partes contractuales, deberían hacer que falle para especificar un término que es relevante para la disputa. 
La tabla 4 enlista cada término indagado en la EULA y como este es puntuado para propósitos de compradores moderados versus vendedores parcializados. Para cada termino esto es más (menos) en favor del comprador que la regla relevante preestablecida, yo añado (sustraigo) un punto. Para cada término que falte o este especificado, pero sustantivamente conecta con la regla preestablecida. No añado nada. Después de todos los términos que han sido considerados, la agrupación representa un simple índice parcializado. Las sumas altamente negativas indican contratos que son relativamente más inclinados hacia el vendedor.

El mejor puntaje de un contrato a favor del vendedor es de -17. Más positivo indica relativamente más contratos a favor del comprador. El mejor puntaje de un contrato a favor del comprador es de +6 . Mientras en crudo, esta metodología es objetiva y no requiere información sobre las preferencias de los compradores.

Recibido: $12 / 07 / 2017$

Aceptado: 29/11/2017

Prof. Florencia Marotta-Wurgler: Profesora de Leyes de la Universidad de New York.

Correo electrónico: wurglerf@exchange.law.nyu.edu 